\title{
Face Recognition Stationed on DT-CWT and Improved 2DPCA employing SVM Classifier
}

\author{
Deepshikha Bhati \\ Computer Science \& Engineering \\ ABES Engineering College \\ Ghaziabad, India
}

\begin{abstract}
Wavelet Transform is basically used for magnitude depletion. It is used for axing the proportion of picture. Including good multi-resolution and multi-scale analysis, wavelet transform also has the propensity of denoting local signal attribute by using the high and low pass filtering, image can be decomposed into divergent scales of approximation components. But in wavelet transform, the higher decomposition layers will lost a lot of information, by which reduce the recognition rate. 2DPCA is a sort of image extraction method deal directly with image data and does not need dimension reduction. It is undertaking image data without step of vectorization. However 2DPCA algorithm reduces the computational complexity, it takes up more storage space. In that paper proposed an advanced technique which is stationed on dual-tree complex wavelet transform and improved 2DPCA employing SVM classifier in order to give higher coherent recognition rate. The experimental results on ORL and YALE face databases shows that the proposed method improves the performance of face recognition with respect to exiting techniques.
\end{abstract}

\section{Keywords}

Face recognition, PCA, 2DPCA, Improved 2DPCA, DT-CWT , SVM.

\section{INTRODUCTION}

There are three prime aspects included in face recognition image preprocessing, feature extraction and classification recognition [1]. Many algorithms on face recognition have been put forward by the researchers [2]. One of them was PCA algorithm which converts the two- dimensional face image matrix into one- dimensional vector image and will provide high dimension image vector, due to this feature extraction amount of calculation becomes lengthy and the rate of recognition is not ideal [26].

Then, the researchers came up with limitation of PCA algorithm that rate of recognition is not ideal and new method proposed stationed on 2DPCA [3] [4].hey come up with method stationed on 2DPCA [3] [4]. This method does not desideratum to alter he image matrix into one dimensional vector in advance. It directly calculates image covariance matrix eigenvector. Although the computing complication reduces, the method is extracted by row compression gimmick of image matrix. Recognition rate is not high because correlation in the row still remains, however it only eliminates the correlation of image matrix in the column [26].

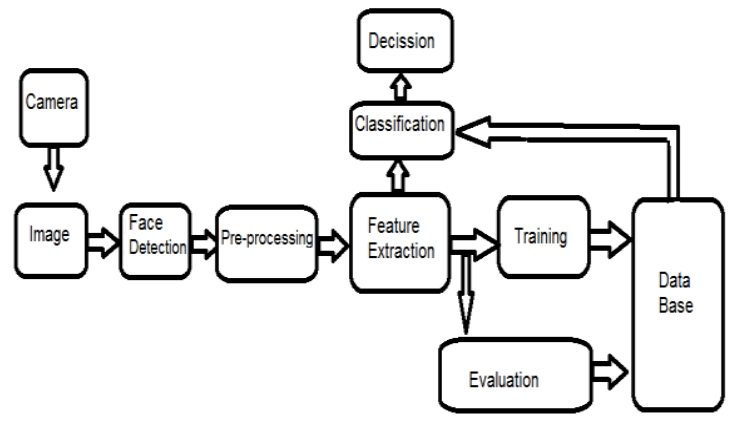

Fig 1: Face recognition system

So, find a way to consider correlation of the images in the rows and columns simultaneously, and it can achieve higher recognition rate. Face recognition method stationed on improved 2DPCA is proposed. It can remove the correlation of the images in the rows and columns simultaneously. It can deal with the image data directly and do not need the evolution of vectorization. Improved 2DPCA reduces the computational complexity [26]. The improvement in the recognition rate comes with a disadvantage to the stash arena (utilization is more) [5]. Using the dual-tree complex wavelet transform (DT-CWT) during the image processing is due to its attractive properties: approximate shift- invariance, orientation selectivity, efficient computation [6]. In [7], the first level DTCWT is employed to transform facial image to frequency domain, before SVM. In [8], [9], the 4-level DT-CWT is performed on facial image, and the high frequency components of all four levels are used to construct the representation. However, they just simply utilize DT-CWT and do not intensively study the performance of DT-CWT for face representation under different contrast, predominantly shift and ornamentation. Also, their depiction are highly unessential and occupy lots of memory, which brings problems for dimension reduction, it is intractable to choose the optimal range of principal components.

So this paper proposes face recognition mechanism stationed on DT-CWT and improved 2DPCA [10]. Firstly, it uses DTCWT for magnitude diminution. Then it uses the improved 2DPCA for feature extraction. Finally, classification recognition is lugged out by using the support vector machine (SVM) method [26].

\section{DT-CWT}

The 1-D DT-CWT is carry out using two alarmingly sampled real DWTs $\varphi_{h}(t)$ and $\varphi_{g}(t)$ in parallel on same data to produce the absolute and imaginative parts of complex wavelet 
$\varphi(t) \triangleq \varphi_{h}(t)+j \varphi_{g}(t)\left(j^{2}=-1\right) \cdot \varphi(t)$ is approximately analytic (i.e. $\varphi_{h}(t)$ is proximately the Hilbert transmute of $\left.\varphi_{g}(t)\right)$, which brings proximately magnitude shift invariance of its wavelet coefficients [11][27].

The 2-D DT-CWT is characterized by six wavelets: $\varphi^{1}(x, y)=\phi(x) \varphi(y), \varphi^{2}(x, y)$

$$
=\varphi(x) \varphi(y), \varphi^{3}(x, y)=\varphi(x) \phi(y)
$$

And their half-conjugate partners

$\varphi^{4}(x, y)=\varphi(x) \phi(y), \varphi^{5}(x, y)=$ $\varphi(x) \varphi(y), \varphi^{6}(x, y)=\phi(x) \varphi(y)$ Where $\quad \varphi(x) \triangleq$ $\varphi_{h}(x)+j \varphi_{g}(x)$ and $\phi(x) \triangleq \phi_{h}(x)+j \phi_{g}(x)$ are the 1-D complex wavelet function and scale function, respectively.

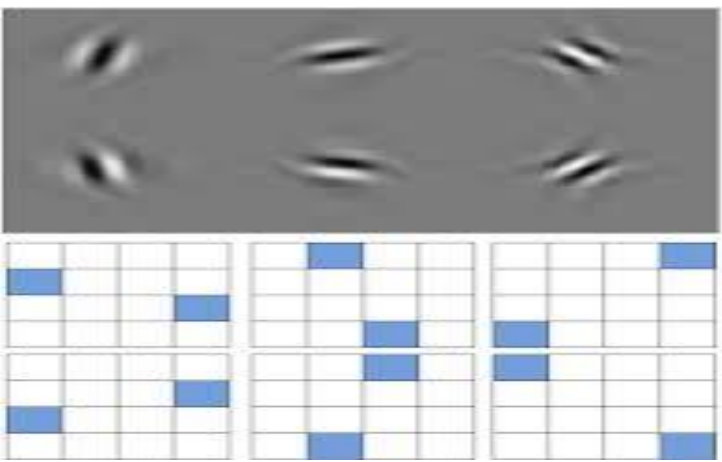

Fig 2: Two-dimensional dual-tree complex wavelets in spatial domain and their (idealized) support of the spectrum in 2-D frequency plane, oriented

at $-75^{\circ},-45^{\circ},-15^{\circ}, 15^{\circ}, 45^{\circ}, 75$, respectively. [21][27]

All the $\varphi^{k}(x, y)(1 \leq k \leq 6)$ give six band pass sub bands of tangled coefficients at each and every level, which are robustly dispose at angles of $-75^{\circ},-45^{\circ},-15^{\circ}, 15^{\circ}, 45^{\circ}, 75^{\circ}$, respectively, as shown in Fig. 2. The 2-D DT-CWT succeeds in isolating identical emplacement. Promptly, we review the expression of $\varphi^{2}(x, y)$ as

$$
\begin{gathered}
\varphi^{2}(x, y)=\left[\varphi_{h}(x)+j \varphi_{g}(x)\right]\left[\varphi_{h}(y)+j \varphi_{g}(y)\right] \\
=\varphi_{h}(x) \varphi_{h}(y)-\varphi_{g}(x) \varphi_{g}(y) \\
+j\left[\varphi_{g}(x) \varphi_{h}(y)+\varphi_{h}(x) \varphi_{g}(y)\right] .
\end{gathered}
$$

It shows that the real/imaginary part of $\varphi^{2}(x, y)$ can be implemented by subtraction/addition of two 2-D separable DWT in parallel and only requires four-fold computation of 2D DWT and the magnitude coefficients are with a redundancy factor of only $2: 1$. Similar calculations are also true for the rest five 2-D complex wavelets.

The properties of approximately good directional selectivity, magnitude shift-invariance, limited redundancy, efficient linear computation can be harnessed to compute accurate estimates of the geometrical structure in images (the details can be commence in [6][27]). Thus, DT-CWT is natural for analyzing images, and has been pre-owned in some fields of image/video processing, such as image/ video denoising [12], image segmentation [13], pattern recognition [14] and facial gimmick eradication [15][27].

\section{IMPROVED 2DPCA}

Given by image A, an $m \times n$ random matrix, $X \in R^{n \times d}(n \geq d)$ is a column vector orthogonal matrix,

through linear transformation $\mathrm{Y}=\mathrm{AX}$, A represents to the image matrix $\mathrm{X}$, which will produce an $\mathrm{m} \times \mathrm{d}$ projection attributes vector $Y$ [16][26], with the projection samples always discrete degree as the criterion function $\mathrm{J}(\mathrm{X})$ can find ideal projection matrix $\mathrm{X}$ :

$$
J(X)=\operatorname{tr}\left(S_{X}\right)
$$

The $S_{X}$ is the covariance matrix projection matrix $\mathrm{Y}, \operatorname{tr}\left(S_{X}\right)$ is a trace of $S_{X}$

$$
\begin{aligned}
& J(X)=\operatorname{tr}\left\{E\left\lfloor(A X-E(A X))(A X-E(A X))^{T}\right\rfloor\right\} \\
& =\operatorname{tr}\left\{X^{T} E\left\lfloor(A-E A)^{T}(A-E A)\right\rfloor X\right\}
\end{aligned}
$$

Assuming that there are $\mathrm{M}$ training face samples, using $A_{1}(1=1,2 \ldots M)$ to express, an $\mathrm{m} \times \mathrm{n}$ matrix, the average image is [26] :

$$
\overline{\mathrm{A}}=\frac{1}{M} \sum_{1=1}^{M} A_{1}
$$

The $\mathrm{G}$ is estimated to be:

$$
G=\frac{1}{M} \sum_{1=1}^{M}\left(A_{1}-\overline{\mathrm{A}}\right)^{T}\left(A_{1}-\overline{\mathrm{A}}\right)
$$

The largest eigenvalues $X_{1}, X_{2} \ldots X_{d}$ which the former $\mathrm{d}$ eigenvectors of matrix $\mathrm{G}$ corresponding to consist projection matrix $X_{o p t}$. Namely, $X_{o p t}=\left[X_{1}, X_{2} \ldots X_{d}\right]$ is the optimal value, after the projection, matrix $X_{o p t}=\left[X_{1}, X_{2} \ldots X_{d}\right]$ is obtained, it can carry out gimmick eradication and allotting the face image, for given image A [26].

$$
Y_{1}=A X_{1}(1=1,2 \ldots d)
$$

(4)

Getting a set of characteristics vector $L=\left[Y_{1}, Y_{2} \ldots Y_{d}\right]$, it is called the principal constituent vector of A image. In fact, for (3) might as well make [26] :

$$
A_{1}=\left[\left(A_{1}{ }^{(1)}\right)^{T}\left(A_{1}{ }^{(2)}\right)^{T} \ldots\left(A_{1}{ }^{(m)}\right)^{T}\right]^{T}
$$

$$
\overline{\mathrm{A}}=\left[\left(\overline{\mathrm{A}}^{(1)}\right)^{T}\left(\overline{\mathrm{A}}^{(2)}\right)^{T} \ldots\left(\overline{\mathrm{A}}^{(m)}\right)^{T}\right]^{T}
$$

(6)

At this point, $\mathrm{A}_{1}{ }^{(\mathrm{i})}$ and $\overline{\mathrm{A}}^{(\mathrm{i})}$ are respectively the $A_{1}$ and $\overline{\mathrm{A}}$ of the i line vector, then

$$
G=\frac{1}{M} \sum_{\substack{1 \leq 1 \leq M \\ 1 \leq i \leq m}}\left(A_{1}{ }^{(i)}-\overline{\mathrm{A}}^{(i)}\right)^{T}\left(A_{1}{ }^{(i)}-\overline{\mathrm{A}}^{(i)}\right)
$$

Now (5) \& (6) as well be substitute to

$A_{1}=\left[\left(A_{1}{ }^{(1)}\right)\left(A_{1}{ }^{(2)}\right) \ldots\left(A_{1}{ }^{(m)}\right)\right]$

(8)

$\overline{\mathrm{A}}=\left[\left(\overline{\mathrm{A}}^{(1)}\right)\left(\overline{\mathrm{A}}^{(2)}\right) \ldots\left(\overline{\mathrm{A}}^{(m)}\right)\right]$

(9) 
Here, $\mathrm{A}^{1}(\mathrm{j})$ and $\overline{\mathrm{A}}(\mathrm{j})$ are respectively the $A_{1}$ and $\overline{\mathrm{A}}$ the $\mathrm{j}$ column vector, then based on direction of column of image $G$ can be describe as the covariance matrix [26] :

$$
G=\frac{1}{M} \sum_{\substack{1 \leq 1 \leq M \\ 1 \leq j \leq m}}\left(A_{1}^{(j)}-\overline{\mathrm{A}}^{(j)}\right)\left(A_{1}^{(j)}-\overline{\mathrm{A}}^{(j)}\right)^{T}
$$$$
\text { (10) }
$$

Where $P \in R^{m \times q}$ is a column vector matrix which is perpendicular to each other. A projects to the image matrix $P$, produce a size of matrix $B(q \times n)[26]$.

$$
B=P^{T} A
$$

The same, it can calculate the former $\mathrm{q}$ maximum characteristic vale of characteristics vectors $P_{1}, P_{2} \ldots P_{q}$. To get the ideal projection matrix $P_{\text {opt }}=\left\lfloor P_{1}, P_{2} \ldots P_{q}\right\rfloor$. So we can use the ideal projection matrix $\mathrm{X}$ and $\mathrm{P}$ to improve the improved 2DPCA algorithm. The image-matrix of A project to $\mathrm{X} \& \mathrm{P}$ at the same time, that will generate matrix $\mathrm{U}(\mathrm{q} \times \mathrm{d})$ [26].

$$
U=P^{T} A X
$$

Matrix $U$ is called characteristics matrix. The training sample image $A_{1}(1=1,2 \ldots M)$ projects on the $\mathrm{X}$ and $\mathrm{P}$ at the equivalent time to get the tutelage sample characteristics matrix $U_{1}(1=1,2 \ldots M)$. Then to elect a testing image A to obtain its characteristics matrix $U$ by (12), and then to use the SVM as a classifier to classify recognition [26]

\section{SUPPORT VECTOR MACHINE}

SVM is one of the best method for classification which is used frequently now a days. Hyper planes can classify data, which is either linearly separable or non-linearly separable. For nonlinearly detachable data it uses kernel. Further steps are described as follows. [17]

\section{SVM Algorithm}

Given training set

$$
\begin{gathered}
S=\left\{\left(X_{1}, Y_{1}\right),\left(X_{2}, Y_{2}\right), \ldots,\left(X_{m}, Y_{m}\right) \mid\left(X_{i}, Y_{i}\right)\right. \\
\left.\in \mathfrak{R}^{n} \times\{+1,-1\}\right\}
\end{gathered}
$$

1. Choose a map $\phi: \mathfrak{R}^{n} \rightarrow F$, which maps $\mathrm{X}_{\mathrm{i}}$ to a higher dimensional feature space. (Solves problem that $\mathrm{X}$ might not be linearly detachable in authentic arena.)

2. Choose a cheap-to-compute kernel function

$$
K(X, Z)=\phi(X) \cdot \phi(Z)
$$

(Solves problem that is altitudinous dimensionality spaces, dot products are very expensive to computing.)

3. Map all the $X_{i}$ 's to gimmick arena $F$ by measure $\phi$ $\left(\mathrm{X}_{\mathrm{i}}\right)$.

4. Assign quadratic prearrange measure (using the kernel activity $\mathrm{k}$ ) to find $\left(\mathrm{w}, \mathrm{w}_{\mathrm{o}}\right)$, such that

$$
w=\sum_{i} \alpha_{i} \phi\left(X_{i}\right)
$$

Where the $\phi\left(\mathrm{X}_{\mathrm{i}}\right)$ 's are support vectors, the $\alpha_{\mathrm{i}}$ 's are coefficients, and $\mathrm{w}_{\mathrm{o}}$ is a threshold, such that $\left(\mathrm{w}, \mathrm{w}_{\mathrm{o}}\right)$ is the hyper plane maximizing the margin of $\mathrm{S}$ in $\mathrm{F}$.
5. Now, inured a different instance, $\mathrm{x}$, find the classification of

$$
\begin{gathered}
\mathrm{x} \text { by computing } \\
\left.\operatorname{class}(x)=\operatorname{sgn}\left(\sum \alpha_{i}\left(\phi(x) \cdot \phi\left(x_{i}\right)\right)+w_{o}\right)\right) \\
\left.=\operatorname{sgn}\left(\sum \alpha_{i} k\left(x, x_{i}\right)+w_{o}\right)\right)
\end{gathered}
$$

\section{PROPOSED FACE RECOGNITION ALGORITHM}

This article originates from the perspective of image preprocessing. The two groups in which images are branched into are, the tutelage facsimile, and the testing images. Firstly, it proportionately processes the training and testing images with "dual-tree complex wavelet transform" to get the different scales of wavelet sub images then determine all the appropriate division of the images to take the place of the native image as the training images [26]. It acquires certain scale of the image and deduce the image dimensions, also eliminates tangential details, and use the improved 2DPCA method to further obtain the face feature information [18][26]. Ultimately, by employing support vector machine classification method for classification and recognition, in order to verify the effect of image feature extraction [19][26].

The dual-tree wavelet transform treated as the image preprocessing method combined with improved 2DPCA algorithm to carry on image feature extraction principle is as follows [26]:

The training process is as follow:

1. To select the appropriate wavelet base and decomposition layer, it is aligning out on the training image with DT-CWT for the image of face image database, and acquire the different scales of wavelet sub-images. After the DT-CWT, reducing the dimensions of the image, low frequency components replace the original face to make the sample matrix [20][26].

2. By using the improved 2DPCA for training images to extract image feature. To elect a few eigenvectors that corresponding to characteristic value as the ideal projection matrix[26].

The testing process is as follow:

1. The face sample matrix maps to the projection grid, and obtain the attribute of the tutelage fragment coefficients to constitute the tutelage fragment space [26].

2. The same as the training process, to carry out on the test image with wavelet transform, and obtain the face samples. Also it can use the improved 2DPCA method for feature extraction, and select a number of eigenvectors that corresponding to eigenvalue to constitute the ideal projection grid. And at the ideal projection matrix to map so as to gain coefficient of the characteristics of the testing sample to constitute testing sample space [26].

3. After feature extraction, it take face feature into the classifier for identification. When a face sample comes into classifier, sample should belong to a type of space type in order to actuate the samples of 
unknown categories. To identify and classify, SVM classification method is used. Through the calculation of test fragment and each training sample feature to select test sample which is closest to sample for test sample category [26].

\section{EXPERIMENTAL RESULT}

Proposed algorithm validate by using the standard face databases that are ORL Face Database and Yale Face Database. Then partition the database into following subsets and further use it in training and testing phase. In proposed approach used Leave-one-out method that randomly select an image from database, left images used in training phase and rate of recognition was calculated. For implementation, of proposed algorithm used MATLAB R2011a [28].

\subsection{Face Database}

\subsubsection{ORL Face Database:}

Olivetti Research Laboratory face database is used which contain 400 images of 40 peoples, where each person has 10 pictures in different poses [21][28]. This database consist different types of facial expressions of different persons at different moments by using some effects and deviations like lightening, with spectacles or without, beard or beardless of both females and males. It subsist grey-scale images in $92 \times 112$ pixels as mentioned in Fig 3.

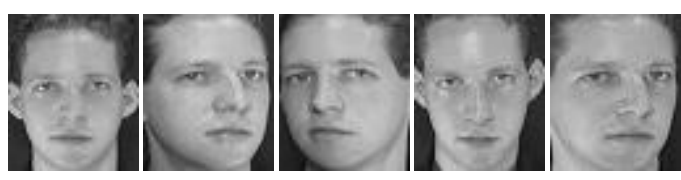

Fig 3: Display a sample set of ORL Face Database (only 5 pose out of 10)[28]

\subsubsection{Yale Face Database:}

In this database, there are 165 grey-scale images of 15 persons in GIF format [22]. Database included 11 images per subject; each image has distant facial statement like lightening effects, with spectacles or without, different emotions (like sad, happy, wink, sleepy, surprising) and normal. It subsist grey-scale images in $320 \times 243$ pixels as mentioned in Fig 4.

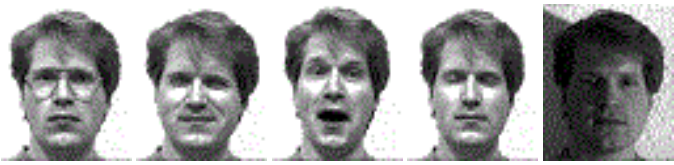

Fig 4: Display a sample set of Yale Face Database (only 5 pose out of 11) [28]

\subsection{Classification}

In this paper used SVM Classifier to classify the faces. It is very recent and effective classification method. In this paper trained features (data) after preprocessing with DTCWT and Improved 2DPCA. As discussed above that SVM classify the data using hyperplanes, and it normally classify data into two classes only, but used multi SVM method that classify data more than two classes using the divide and conquer phenomena.

\subsection{Result and Analysis}

In proposed approach used Leave-one-out method that randomly select an image from database, left images used in training phase and rate of recognition was calculated. For the implementation of proposed algorithm used MATLAB R2011a. Then, resulted results are compared with different preexisting methods which using same database. In Table 1 demonstrate the result of the proposed algorithm along with comparing with pre-existing algorithms. Proposed hybrid algorithm, that combine DTCWT, Improved 2DPCA and SVM (Support Vector Machine). Proposed method has highest recognition rate at dimension reduction of 50X50 ( ORL = $99.54 \%$, YALE $=98.7 \%$ ).

Table 1: Comparison made within proposed and different pre-existing methods which using same database

\begin{tabular}{|c|c|c|c|}
\hline \multirow[t]{2}{*}{ Strategy } & \multirow[t]{2}{*}{ Method } & \multicolumn{2}{|c|}{ Recognition Rate (\%) } \\
\hline & & $\begin{array}{c}\text { ORL } \\
(92 \times 112)\end{array}$ & $\begin{array}{c}\text { YALE } \\
(320 \times 243)\end{array}$ \\
\hline \multirow{3}{*}{$\begin{array}{l}\text { Leave- } \\
\text { one-out } \\
\text { method }\end{array}$} & DTCWT+SVM & 99.54 & 98.5 \\
\hline & $\begin{array}{c}\text { Improved } \\
\text { 2DPCA+SVM }\end{array}$ & 99.87 & 98.6 \\
\hline & $\begin{array}{c}\text { DTCWT+ } \\
\text { Improved } \\
\text { 2DPCA +SVM } \\
(\mathbf{5 0 X 5 0 )}\end{array}$ & 99.8 & 98.7 \\
\hline
\end{tabular}

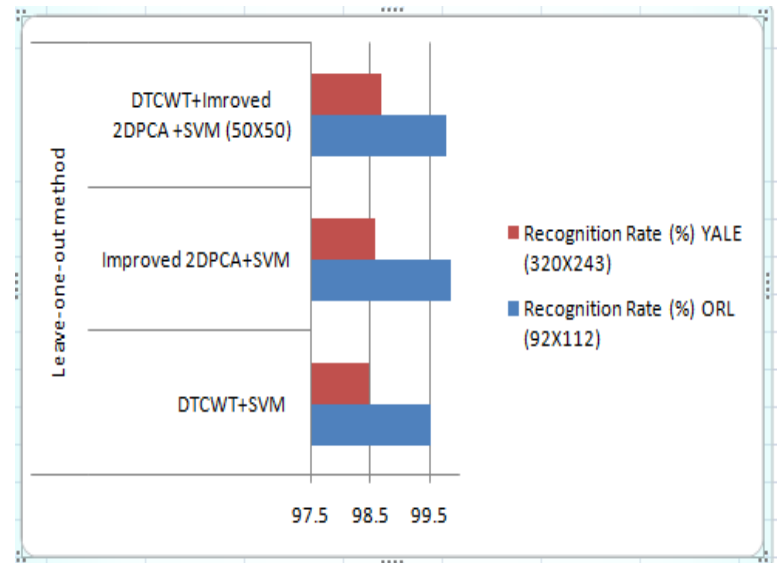

Fig 5: Bar-graph representation of compared results of proposed method with different pre-existing methods which using same database

In Table 2 the recognition rate is carried out under different dimension. The recognition rate of Yale dataset at $15 \mathrm{X} 15$ dimensions is $93.33 \%$ and at 20X20 dimensions is $93 \%$. In case of ORL Dataset the recognition rate is far better than YALE Dataset i.e. at 15X15 dimensions the recognition rate is $95.16 \%$ and at $20 \mathrm{X} 20$ dimensions is $99.12 \%$.

Table 2: Recognition Rate (\%) under different dimension

\begin{tabular}{|l|c|c|}
\hline \multicolumn{1}{|c|}{ Method } & \multicolumn{2}{c|}{ Recognition Rate (\%) } \\
\hline Dimension & $15 X 15$ & $\mathbf{2 0 X 2 0}$ \\
\hline YALE dataset & $\mathbf{9 3 . 3 3}$ & $\mathbf{9 3}$ \\
\hline ORL dataset & $\mathbf{9 5 . 1 6}$ & $\mathbf{9 9 . 1 2}$ \\
\hline
\end{tabular}

\section{CONCLUSION}

From the various comparative analyses among various approaches, recognize in order that face recognition stationed on dual-tree complex wavelet transform and improved 2DPCA employing SVM classifier can enhance the recognition rate. To reduce dimension treat DT-CWT as a preprocessing and it is better and efficient to elicitation facial features robust facing the divergence of shift and illumination, basically DT-CWT is a beneficial intensification for the ancestral real wavelet transform and used improved 2DPCA approach in order to remove image correlations in the rows and columns in simulating manner. It basically overcome plentiful shortcomings, for instance taking up numerous 
storage space and high computational complexity. And employing SVM classifier which shows the best efficiency till now for classification. The proposed approach devise recognition rate. In future work this method can be enhanced by using different databases with multiple subjects and also focused on time complexity.

\section{REFERENCES}

[1] Yang Shaohua, Pan Chen, Huang Wenyan. Using Image Preprocessing Method Improved 2DPCA Face Recognition Performance, Journal Of Lanzhou University Of Technology, 2008, 02,34(2),pp. 82-87.

[2] Turk M. Pentland A. Eigen faces For Recognition, Journal of Cognitive Neuroscience, 1991, 3(1), pp. 71-86.

[3] Yang J, Zhang D, Yang J Y. Two- Dimensional PCA: A New Approach To Appearance- Based Face Representation And Recognition, IEEE Trans. on Pattern L Machine Intel. L, 2004, 26(1), pp. 131-137.

[4] Zhang Daoqiang, Zhou Zhihua. Two- Directional TwoDimensional PCA for efficient Face Representation and Recognition, Neurocomputing, 2005, 69(3), pp.224-231.

[5] Yang Wankou, Ji Shanbing, Ren Mingwu. Based on The Enhanced 2 Dimension Feature Extraction Methods of Principal Component Analysis and its Application in Face Recognition, Chinese Journal of Image, 2009(2), pp. 227-232.

[6] I. W. Selesnick, R.G. Baraniuk, and N. G. Kingsburg, The dual- tree complex wavelet transform- a coherent framework for multiscale signal and image processing, IEEE Signal Process. Mag., vol. 22, no. 6, pp. 123-151, Nov. 2005.

[7] G. Y. Zhang, S. Y. Peng, and H. M. Li, Combination of dual-tree complex wavelet and svm for face recognition, in Proc. Ibt Conf. Machine Learning and Cybernetics, 2008, vol 5, pp. 2815-2819.

[8] Y. H. Sun and M. H. Du, Dt-CWT feature combined with onpp for face recognition, in computational Intelligence and Security. Berlin/ Heidelberg, Germany: Springer, 2007, vol. 4456, pp. 1058-1067.

[9] Z. M. Huang, X. H. Zhang, and D. Yang. Face recognition based on dual-tree complex wavelet feature, J. Comput. Appl. , vol. 27, no. 5, pp. 1135-1137, 2007.

[10] Wang YuDe Zhao HuanLi, Xue NaiYu. Face feature Extraction And Recognition Algorithm Based On the Block OF The Wavelet Transform And 2DPCA, Infrared And Laser Engineering 2012. Pp.3119-3121.

[11] N. G. Kingsbury, Complex wavelets for shift invariant analysis and filtering of signals, Appl. Comput. Harmon. Anal. , vol. 10, no. 3, pp. 234-253, 2001.

[12] H. Rabbani and M. Vafadust, Image/video denoising based on a mixture of laplace distributions with local parameters in multiimensional complex wavelet domain, Signal Process. , vol. 88, pp. 158-173, 2008.

[13] E. H. S. Lo, M.R. Pickering, M. R. Frater, and J. F. Arnold. Image segmentation using invarient texture features from the double dyadic dual-tree complex wavelet transform, in Proc. IEEE Int. conf. Acoustics, speech and signal Processing, Apr. 2007, vol. 1, pp. I609-I-612.
[14] G. Y. Chen, T. D. Bui, and A. Krzyzak,Invarient pattern recognition using radon, dual- tree complex wavelet and fourier transforms , Pattern Recognition., vol. 42, no. 9, pp. 2013-2019, sep. 2009.

[15] T. Celik, H. Ozkaramanli, and H. Demirel.Facial feature extraction using complex dual- tree wavelet transform, comput. Vis. Image Understand. , vol. 111, pp. 229-246, 2008.

[16] Li Tong. Research. On Human Face Recognition Method Based On The Improved 2DPCA, Journal of Chongqing Industry And Commerce University, 2012. Pp. 46-48.

[17] Cortes.C., Vapnik.V. (1995).Support- Vector Networks, Machine Learning 20(3): 273. Doi:10.1007/BF00994018.

[18] Han XiaoCui. An Improved 2DPCA Face Recognition Method, Computer Engineering And Application, 2010 46(25). Pp.185-187.

[19] Li WuJun, Wang Chongjun, Zhang Wei, Face Recognition technology, Journal Of Pattern Recognition And Artificail Intelligence, 2006, 19(1). Pp. 58-66.

[20] Wan MingHua, Liu Zhonghua Jin Zhong. A Face Recognition Method Based On The 2DPCA And SVM, Journal Of Communication Technology. 2009, 42(5), pp. 100-102.

[21] ORL database: http://www.camorl.co.uk.

[22]Yale Face Database: http://cvc.yale.edu/projects/yalefaces

[23]M.Pal ， D.Bhati, B.kaushik, H.Banka. Solving Classification Problem Using Reduced Dimesion and Eigen Structure in RSVM, International Journal of Computer Applications( 0975-8887) volume 117-no.21, may 2015 .

[24] D.Bhati, V.Gupta. Survey- A Comparative Analysis of Face Recognition Technique, International Journal of Engineering Research and Genral Science Volume 3, issue 2, March- April , 2015 ISSN 2091-2730.

[25] Aili Wang, Na Jiang and Yuan Feng. Face Recognition Based on Wavelet Transform and Improved 2DPCA, 2014 Fourth International Confrence on Instrumentation and Measurement, computer, communication and control, 2014

[26] Chao- Chun Liu, and Dao-Qing Dai.Face Recognition using Dual- Tree <newline/> Complex Wavelet Features, IEEE Transactions on Image processing, 2009.

[27]Dandpat, Swarup Kumar, and Sukadev Meher. Performance improvement for face recognition using PCA and two dimensional PCA, 2013 International confrence on computer communication and informatics, 2013. 